\title{
Preliminary study on total protein extraction methods from Enterococcus faecalis biofilm
}

\author{
W. Chen ${ }^{1}$, J. Liang ${ }^{2}$, Z. He ${ }^{3}$ and W. Jiang ${ }^{2}$ \\ ${ }^{1}$ Department of Stomatology, Eye \& ENT Hospital of FuDan University, \\ Shanghai, China \\ ${ }^{2}$ Department of Endodontics and Operative Dentistry, \\ Shanghai Key Laboratory of Stomatology, Shanghai Jiao Tong University, \\ Shanghai, China \\ ${ }^{3}$ Shanghai Research Institute of Stomatology Ninth People's Hospital, \\ School of Medicine, Shanghai Jiao Tong University, Shanghai, China \\ Corresponding authors: W. Jiang / J.P. Liang \\ E-mail: jiangwei1981@hotmail.com / liangjpshanghai@126.com
}

Genet. Mol. Res. 15 (3): gmr.15038988

Received July 20, 2016

Accepted August 1, 2016

Published August 30, 2016

DOI http://dx.doi.org/10.4238/gmr.15038988

Copyright $(2016$ The Authors. This is an open-access article distributed under the terms of the Creative Commons Attribution ShareAlike (CC BY-SA) 4.0 License.

\begin{abstract}
Enterococcus faecalis is the major pathogen of postendodontic disease and refractory periapical periodontitis, and recent research on this species has focused on its pathogenicity. E. faecalis most often causes disease in the form of a biofilm, and total protein expression shows a strong association with its virulence. Therefore, the purpose of our study was to explore different methods of extracting the total proteins of the E. faecalis (ATCC 33186 standard strain) biofilm. The total proteins in the biofilm were extracted using an ultrasonication method with varied parameters, including duration, amplitude setting, period, and duty cycle. After the optimal conditions of ultrasonication were determined based on the protein profile from sodium dodecyl sulfate-polyacrylamide gel electrophoresis analysis, the total protein
\end{abstract}

Genetics and Molecular Research 15 (3): gmr.15038988 
content in the biofilm was detected using the bicinchoninic acid assay, Bradford Coomassie brilliant blue assay, and Lowry assay, and the results were compared and analyzed. The parameters for the optimal conditions of ultrasonication were as follows: a processing duration of $2 \mathrm{~min}$, amplitude setting of $20 \%$, and ultrasonication period of $4 \mathrm{~s}$ at a $50 \%$ duty cycle. The total protein content was $2299.1 \mathrm{mg} / \mathrm{dish}$ when measured by the bicinchoninic assay, $3793.8 \mathrm{mg} / \mathrm{dish}$ when measured by the Bradford Coomassie brilliant blue assay, and $1858.0 \mathrm{mg} / \mathrm{dish}$ when measured by the Lowry assay. These results demonstrate that the Bradford Coomassie brilliant blue assay is a simple and feasible method for use in detecting the total protein content in a bacterial biofilm.

Key words: Enterococcus faecalis; Biofilm; Ultrasonication; Protein

\section{INTRODUCTION}

Bacterial invasion plays an important role in the occurrence and developmental processes of endodontic and periapical diseases. The existence of bacteria in the root canal system after a filling procedure is one of the most important contributors to the failure of a root canal treatment. In particular, the Enterococcus faecalis present in the root canal mainly exists in the form of a biofilm. This bacterium can survive and cause reinfection at different temperatures $\left(10^{\circ}-45^{\circ} \mathrm{C}\right)$, under different osmotic pressures, in highly acidic or alkaline environments, in the presence of antibiotics, and under other harsh conditions. During the formation of the biofilm, E. faecalis can secrete large amounts of extracellular polymers such as proteins and polysaccharides, which provide protection (Nair, 2006; Siqueira and Rôças, 2008; Gu et al., 2010; Saber and El-Hady, 2012). Therefore, analysis of the biochemical composition of a bacterial biofilm can contribute to the development of new techniques to prevent biofilm infection. In this study, the E. faecalis standard strain ATCC 33186 was used to form a biofilm and the total protein was extracted by ultrasonication. The optimal conditions of the ultrasonication procedure were determined, and the total protein content in the biofilm was detected using three different methods: the bicinchoninic acid (BCA) assay, Bradford Coomassie brilliant blue assay, and Lowry assay. The obtained results were compared to identify a feasible and simple method for determining the total protein content in a bacterial biofilm.

\section{MATERIAL AND METHODS}

\section{Main materials and strain}

The main materials and strain used in this study included: E. faecalis standard strain ATCC 33186; trypticase soy yeast extract broth (TSB; Hangzhou Tianhe Microorganism Reagent Co. Ltd., Hangzhou, China; a sonicator (VCX130, Sonics, Newtown, Connecticut, USA), spectrophotometer (UV1601, Shimadzu, Kyoto,Japan), automatic microplate reader (Elx800, BioTek, Winooski,Vermont, USA), small vertical electrophoresis system (Mini-PROTEAN Tetra System, Bio-Rad, Hercules, CA, USA), electrophoresis apparatus (PowerPac Basic, BioRad, Hercules, CA, USA), and G:BOX gel imaging system (Syngene, Cambridge, UK).

Genetics and Molecular Research 15 (3): gmr.15038988 


\section{Preparation of the bacterial biofilm}

The E. faecalis standard strain ATCC 33186 was inoculated into the TSB and cultured at $37^{\circ} \mathrm{C}$ overnight. After the concentration of the culture broth was adjusted to approximately 2 x $10^{9}$ colony-forming units $/ \mathrm{mL}, 80 \mu \mathrm{L}$ culture broth was inoculated into a glass dish containing $20 \mathrm{~mL}$ fresh TSB medium. After the medium and inoculant were cultured for $24 \mathrm{~h}$ to form the biofilm, the supernatant in the dish was discarded. The biofilm was washed three times with sterile phosphate-buffered saline (PBS) to remove the floating bacteria on the surface. The biofilm of the bacterial cells was scraped and collected in a 1.5-mL Eppendorf tube.

\section{Optimization of the ultrasonication conditions for protein extraction from the biofilm}

\section{Influence of ultrasonication duration}

One-milliliter biofilm and $9 \mathrm{~mL}$ PBS buffer were added to a $50-\mathrm{mL}$ centrifuge tube, followed by ultrasonication in an ice bath with different ultrasonication durations: $30 \mathrm{~s}, 1,2,6$, and 10 min. After the ultrasonication, the Bradford Coomassie brilliant blue assay was applied to detect the biofilm protein content for the different ultrasonication durations. We repeated the experiment three times. The mean optical density at $595 \mathrm{~nm}\left(\mathrm{OD}_{595}\right)$ value was calculated, and the protein concentration after dilution was determined based on the standard curve. The equation for the standard curve was $\mathrm{Y}=0.0016 \mathrm{X}$ with $\mathrm{R}^{2}=0.9993$. The best ultrasonication duration was then selected.

\section{Influence of ultrasound amplitude setting}

Following the same procedures described above, different amplitude settings were applied: 20,60 , and $100 \%$. After the ultrasonication, the same detection method as described above was used to determine the optimal amplitude setting for ultrasonication. We repeated the experiment three times. The mean $\mathrm{OD}_{595}$ value was calculated, and the protein concentration after dilution was determined based on the standard curve. The standard curve equation was $\mathrm{Y}$ $=0.0016 \mathrm{X}$ with $\mathrm{R}^{2}=0.9993$.

\section{Influence of ultrasonication period and duty cycle}

With the same procedures described above, different ultrasonication periods at a $50 \%$ duty cycles were applied: a period of $2 \mathrm{~s}$ at a $50 \%$ duty cycle, a period of $4 \mathrm{~s}$ at a $50 \%$ duty cycle, and a period of $10 \mathrm{~s}$ at a $50 \%$ duty cycle. The total ultrasonication duration remained the same $(2 \mathrm{~min})$. We repeated the experiment three times. The mean $\mathrm{OD}_{595}$ value was calculated, and the protein concentration after dilution was determined based on the standard curve. The standard curve equation was $\mathrm{Y}=0.0016 \mathrm{X}$ with $\mathrm{R}^{2}=0.9993$. After the ultrasonication, the same detection method as described above was used to determine the optimal ultrasonication period and duty cycle combination.

\section{Sodium dodecyl sulfate-polyacrylamide gel electrophoresis (SDS-PAGE) analysis}

Loading buffer was added to the protein samples obtained from the different ultrasonic

Genetics and Molecular Research 15 (3): gmr.15038988 
conditions. After being thoroughly mixed, the samples were heated in a boiling water bath $\left(100^{\circ} \mathrm{C}\right)$ for $3-5$ min while the protein marker was loaded. Using a 5\% stacking gel and a $12 \%$ separating gel, discontinuous vertical slab electrophoresis was performed with the electrophoresis buffer of Tris-glycine buffer ( $\mathrm{pH} 8.3$, containing $0.1 \%$ SDS). Using a DC power supply, the voltage on the stacking gel was adjusted to $50 \mathrm{~V}$, and the voltage on the separating gel was adjusted to $200 \mathrm{~V}$. After the electrophoresis was completed, the electrophoresis cassette was disassembled, and the gel was carefully placed into the Coomassie blue staining solution to soak. The mixture was shaken at room temperature in a mechanical shaker for $30 \mathrm{~min}$. After removing the staining solution, the gel was rinsed with distilled water several times, soaked in the destaining solution, and shaken at room temperature in a mechanical shaker until the protein bands were clearly visible. The destaining solution was replaced when needed to best observe the protein bands, and the image of the electrophoresis gel was captured using the gel imaging system.

\section{Determination of the protein content in the biofilm}

\section{BCA assay}

The biofilm obtained after ultrasonication was diluted with PBS buffer at 1:10. Three $1.5-\mathrm{mL}$ centrifuge tubes were prepared, with $100 \mu \mathrm{L}$ PBS buffer added to one tube as the control and $100 \mu \mathrm{L}$ diluted sample added to each of the remaining tubes. One milliliter BCA Working Solution (BCA Protein Assay Kit, Shanghai Sangon Biotech. Co., Ltd., Shanghai, China) was added to each of the three tubes and mixed quickly. After incubation in a $60^{\circ} \mathrm{C}$ water bath for $30 \mathrm{~min}$, the samples were cooled to room temperature, and the $\mathrm{OD}_{562}$ value of each tube was measured with a spectrophotometer. The mean $\mathrm{OD}_{562}$ value of the two solutions diluted from the same sample was calculated, and the protein concentration after dilution was determined based on the standard curve. The standard curve equation was $\mathrm{Y}=0.0011 \mathrm{X}$ with $\mathrm{R}^{2}=0.9952$. Then, the protein concentration of the sample was calculated according to the following equation: Protein concentration of the sample $(\mathrm{mg} / \mathrm{dish})=$ Protein concentration of the diluted sample $\mathrm{x}$ dilution factor of the sample.

\section{Bradford Coomassie brilliant blue assay}

The biofilm obtained after ultrasonication was diluted with PBS buffer at an appropriate ratio. Three $1.5-\mathrm{mL}$ centrifuge tubes were prepared, with $100 \mu \mathrm{L}$ PBS buffer added to one tube as the control and $100 \mu \mathrm{L}$ diluted sample added to each of the remaining tubes. One milliliter Bradford Reagent (Bradford Protein Assay Kit, Shanghai Sangon Biotech. Co., Ltd.) was added to each of the three tubes and mixed quickly. The samples were allowed to stand at room temperature for $5 \mathrm{~min}$, and the solution was mixed thoroughly by inverse blending 1-2 times during this 5-min interval. The $\mathrm{OD}_{595}$ value of each tube was measured with a spectrophotometer. The mean $\mathrm{OD}_{595}$ value of the two solutions diluted from the same sample was calculated, and the protein concentration after dilution was determined based on the standard curve. The standard curve equation was $\mathrm{Y}=0.0016 \mathrm{X}$ with $\mathrm{R}^{2}=0.9993$. The protein concentration of the sample was then calculated according to the following equation:

Protein concentration of the sample $(\mathrm{mg} / \mathrm{dish})=$ Protein concentration of the diluted sample $\mathrm{x}$ dilution factor of the sample.

Genetics and Molecular Research 15 (3): gmr.15038988 


\section{Lowry assay}

The biofilm obtained after ultrasonication was diluted with PBS buffer at an appropriate ratio. Three $1.5-\mathrm{mL}$ centrifuge tubes were prepared, with $100 \mu \mathrm{L}$ PBS buffer added to one tube as the control and $100 \mu \mathrm{L}$ diluted sample added to each of the remaining tubes. In each of the three tubes, $5 \mathrm{~mL}$ Reagent A (a mixture of reagents A1, A2, and A3 with a volume ratio of 100:1:1, which was prepared on the day of the assay and allowed to stand for $30 \mathrm{~min}$ before use; Lowry Protein Assay Kit, Shanghai Labaide Biotech. Co., Ltd., Shanghai, China) was added, mixed well, and allowed to stand at room temperature for $10 \mathrm{~min}$. Subsequently, 0.5 $\mathrm{mL}$ Reagent $\mathrm{B}$ was added to each tube and mixed quickly. After standing at room temperature for $30 \mathrm{~min}$, the $\mathrm{OD}_{750}$ value of each tube was measured with a spectrophotometer. The mean $\mathrm{OD}_{750}$ value of the two solutions diluted from the same sample was calculated, and the protein concentration after dilution was determined based on the standard curve. The standard curve equation was $Y=0.0005 X$ with $R^{2}=0.9963$. The protein concentration of the sample was then calculated according to the following equation:

Protein concentration of the sample $(\mathrm{mg} / \mathrm{dish})=$ Protein concentration of the diluted sample $\mathrm{x}$ dilution factor of the sample.

\section{Statistical analysis}

SPSS 17.0 statistical software was used for the statistical analysis. Univariate analysis of variance was carried out, with the test significance level set to $\alpha=0.05$.

\section{RESULTS}

\section{Optimal ultrasonication conditions for protein extraction from the biofilm}

\section{Ultrasonication duration}

The extension of the ultrasonication duration is conducive to disrupting the bacteria, which allows for more protein to be extracted. As shown in Table 1, with an increase in the ultrasonication duration, the concentration of protein also increased, but the rate of increase slowed down after 2 min. Therefore, to improve the efficiency of the protein determination, the ultrasonication duration of 2 min was deemed to be appropriate.

Table 1. Effect of ultrasonic times.
\begin{tabular}{l|c|c|c|c|c}
\hline & \multicolumn{5}{|c}{ Time $(\min )$} \\
\cline { 2 - 6 } & 0.5 & 1 & 2 & 5 & 10 \\
\hline Protein concentration $(\mu \mathrm{g} / \mathrm{mL})$ & $246.875 \pm 5.36$ & $330 \pm 7.69$ & $345 \pm 2.38$ & $346.875 \pm 8.02$ & $357.5 \pm 5.57$ \\
\hline
\end{tabular}

\section{Ultrasound amplitude settings}

Different amplitude settings, including 20,60, and 100\%, were applied, which resulted in negligible changes in the protein concentration (Table 2). More heat is generated with an increase in the amplitude setting, which may cause protein damage; therefore, the lowest amplitude setting of $20 \%$ was selected as the optimal amplitude setting in this study.

Genetics and Molecular Research 15 (3): gmr.15038988 
Table 2. Effect of ultrasonic amplitudes.

\begin{tabular}{l|c|c|c}
\hline & \multicolumn{3}{|c}{ Amplitude } \\
\cline { 2 - 4 } & $20 \%$ & $60 \%$ & $100 \%$ \\
\hline Protein concentration $(\mu \mathrm{g} / \mathrm{mL})$ & $317.25 \pm 5.35$ & $348 \pm 2.17$ & $350.625 \pm 3.68$ \\
\hline
\end{tabular}

\section{Ultrasonication period and duty cycle with $20 \%$ amplitude}

With the same total ultrasonication duration, different ultrasonication periods at a $50 \%$ duty cycles were applied: $2 \mathrm{~s}$ at a $50 \%$ duty cycle, $4 \mathrm{~s}$ at a $50 \%$ duty cycle, and $10 \mathrm{~s}$ at a $50 \%$ duty cycle. The protein concentrations after the ultrasonication are shown in Table 3 . When the ultrasonication period was increased to $4 \mathrm{~s}$, the absorbance value was significantly increased, but when it reached $10 \mathrm{~s}$, very little change was observed. Therefore, the condition of an ultrasonication period of $4 \mathrm{~s}$ at a $50 \%$ duty cycle was selected as the optimum in this study.

Table 3. Effect of ultrasonic and interval times.

\begin{tabular}{l|c|c}
\hline Ultrasonic duration $(\mathrm{s})$ & Interval time $(\mathrm{s})$ & Protein concentration $(\mu \mathrm{g} / \mathrm{mL})$ \\
\hline 1 & 1 & $366.25 \pm 5.19$ \\
\hline 2 & 2 & $490.625 \pm 1.05$ \\
\hline 5 & 5 & $493.125 \pm 9.77$ \\
\hline
\end{tabular}

\section{SDS-PAGE analysis}

The protein samples obtained by the above ultrasonication method with different durations, amplitude settings, periods, and duty cycles were subjected to SDS-PAGE analysis. The resultant images from the gel imaging system are shown in Figure 1. The overall performance of the ultrasonication was satisfactory, with a large number of extracted proteins obtained. The electrophoretic bands were evenly distributed throughout the gel and were clearly visible with high resolution. Samples 1-5 in Figure 1 refer to the proteins obtained with different ultrasonication durations. Figure 1A shows that sample 1 displayed less clearly recognizable bands in comparison with samples 2-5, and the bands for the proteins with smaller molecular weights were particularly blurry with low resolution and low concentration. This result suggested that with the increase in ultrasonication duration, the number and intensity of the protein bands also increased, although these increases peaked at a certain level. Samples 6-8 in Figure 1 represent the proteins obtained with different amplitude settings. The overall brightness and resolution of the resultant protein bands for the three samples were not significantly different, except that the bands for the proteins with smaller molecular weights in sample 8 were slightly paler and blurrier than those obtained for the other two samples. This result indicated that the impact of the amplitude settings on the protein extraction was minor (Figure 1B). Samples 9-11 in Figure 1 are the proteins obtained with different ultrasonication periods and duty cycles. The number and intensity of the protein bands for sample 9 were lower than those for samples 10 and 11 . That is, the protein bands were fewer, thinner, and lighter in sample 9, whereas the protein profiles for samples 10 and 11 were not significantly different (Figure 1C). The results of the SDS-PAGE analysis were consistent with the findings in the above exploration for the optimal ultrasonication conditions. Therefore, an ultrasonication duration of $2 \mathrm{~min}$, the lowest amplitude setting of $20 \%$, and an ultrasonication period of $4 \mathrm{~s}$ at a $50 \%$ duty cycle was selected as the optimal condition for ultrasonication.

Genetics and Molecular Research 15 (3): gmr.15038988 


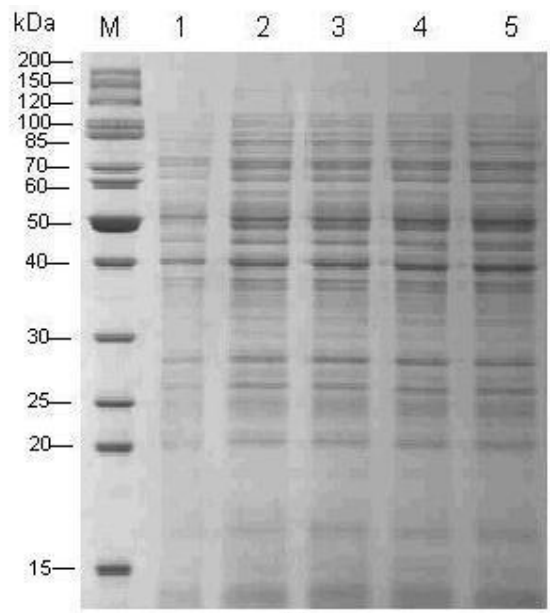

(a)

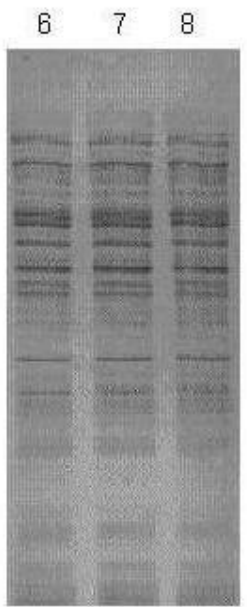

(b)

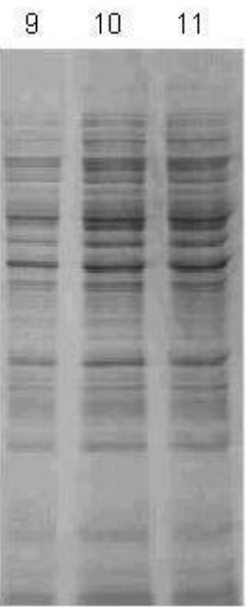

(c)

Figure 1. SDS-PAGE electrophoresis. M: Standard protein marker; a. lanes 1-5 were the protein samples obtained with different ultrasonication duration: lane $1,30 \mathrm{~s}$; lane 2,1 min; lane 3,2 min; lane 4, $5 \mathrm{~min}$; and lane 5, 10 min; b. lanes 6-8 were the protein samples obtained with different amplitude settings: lane 6, 20\%; lane 7, 60\%; and lane $8,100 \%$; c. lanes $9-11$ were the protein samples obtained with different ultrasonication periods and duty cycles: lane 9, an ultrasonication period of $2 \mathrm{~s}$ at $50 \%$ duty cycle; lane 10, an ultrasonication period of $4 \mathrm{~s}$ at $50 \%$ duty cycle; and lane 11 , an ultrasonication period of $10 \mathrm{~s}$ at $50 \%$ duty cycle.

\section{Protein content in the biofilm}

The protein content of the biofilm was determined using different methods. Each experiment was repeated three times, and the calculations of the protein content in each dish of biofilm are shown in Table 4. The protein concentration measured by the Bradford Coomassie brilliant blue assay was the highest ( $3796.8 \mathrm{mg} / \mathrm{dish})$, whereas the concentrations detected by the BCA assay and Lowry assay were lower at $2299.1 \mathrm{mg} /$ dish and $1858.0 \mathrm{mg} /$ dish, respectively. Analysis of variance for the three methods showed a significant difference $(\mathrm{P}<0.05)$. Levels of protein quantified by the Bradford Coomassie brilliant blue assay were significantly greater than those of the $\mathrm{BCA}$ and Lowry assays $(\mathrm{P}<0.05)$. However, there were no significant differences in protein levels quantified with the BCA and Lowry assays.

Table 4. Protein concentration in bilfilm determined by different methods ( $\mu \mathrm{g} / \mathrm{dish}$, means $\pm \mathrm{SD})$.

\begin{tabular}{l|c|c|c}
\hline \multirow{2}{*}{} & \multicolumn{3}{|c}{ Methods } \\
\cline { 2 - 4 } & BCA & Bradford & Lowry \\
\hline Concentration of protein & $2299.1 \pm 178.4$ & $3792.8 \pm 71.6$ & $1858.0 \pm 127.8$ \\
\hline
\end{tabular}

\section{DISCUSSION}

Recent studies have shown that $E$. faecalis is the microbe with the highest detection rate in infected root canals as well as in a root canal that is retreated after the failure of root canal therapy, and is thus considered to be the main pathogen of intractable and secondary root canal infection (Nair, 2006; Siqueira and Rôças, 2008; Gu et al., 2010; Saber and ElHady, 2012). In the root canal, E. faecalis is mainly present in the form of a biofilm, and 
the extracellular polymer of the biofilm has a protective effect for this bacterium. Therefore, investigation of the main biochemical components of the biofilm has important significance for further clarification of the pathogenesis of the disease, risk assessment, and ecological control.

In this study, the total protein was extracted from a biofilm formed with E. faecalis standard strain ATCC 33186, and the protein content of the extracted sample was determined using a simple mechanical method - ultrasonication - under various conditions (Smith et al., 1985; NanoDrop Technical Support Bulletin T010, 2007). Ultrasonic fragmentation is the most common method used for cell disruption, and it is simple and highly efficient. In the ultrasonication process, the ultrasonic vibration at a high frequency is unsynchronized with the vibration of the microbial cells, leading to a localized vacuum environment surrounding the cells. This vacuum environment can cause a cavitation effect on the cell membrane, which generates a mechanical shear stress, turmoil with the vacuole vibration and its subsequent violent implosion, ultimately resulting in disruption of the cell (Jambrak et al., 2008; He et al., 2009). The efficiency of ultrasonic fragmentation depends on the intensity, frequency, and duration of the ultrasonication, as well as the cell type. In the application of ultrasonication, the ultrasonic intensity must be controlled within a certain limit. If the ultrasound intensity is too high, the heat dissipation is very low, and some proteins may be lost through denaturation; if the ultrasound intensity is too low, the cell disruption may be incomplete, and the proteins may not be fully released. By exploration of the impacts of ultrasonication duration, amplitude setting, ultrasonication period, and duty cycle on the experimental results, the following optimal conditions were determined: ultrasonication duration of $2 \mathrm{~min}$, amplitude setting of $20 \%$, and ultrasonication period of $4 \mathrm{~s}$ at a $50 \%$ duty cycle. Under these conditions, the electrophoresis profile for the $E$. faecalis biofilm showed a large number of proteins with clear bands obtained at a high resolution (e.g., sample 10, Figure 1C).

Under these optimal ultrasonication conditions, three different methods (BCA assay, Bradford Coomassie brilliant blue assay, and Lowry assay) were applied to measure the protein content extracted from the biofilm (Brenner and Harris, 1995; NanoDrop, 2009). Comparison of the results among assays showed that the protein content measured by the Bradford Coomassie brilliant blue assay was the highest and the measurement results of the BCA assay and Lowry assay were significantly lower. A possible cause for this difference may be related to the complex metabolic activities for the bacteria to grow, develop, reproduce, and decline during biofilm formation. In this process, a large amount of secreted macromolecule polymers, the adsorbed nutrients, the cytotoxic substances and metabolites, as well as bacterial lysate products such as proteins, polysaccharides, organic acids, enzymes, lipids, and phospholipids are released. Therefore, after ultrasonication, these substances may affect the BCA method by reducing the copper ions into cuprous ions, and might affect the Lowry assay by reducing the phosphomolybdic acid and phosphotungstic acid contents, thus hindering the formation of the colored complex and leading to lower measurable values than the actual contents. By contrast, the Bradford Coomassie brilliant blue assay is based on protein-dye binding; that is, the Coomassie brilliant blue G-250 is brownish-black in an acidic solution and turns blue after binding with proteins, thereby enabling determination of the protein content and avoiding the impact of a redox reaction on color development.

In summary, the present study demonstrated that the Bradford Coomassie brilliant blue assay is a feasible and simple method for the determination of total protein content in bacterial biofilms. Moreover, an ultrasonication duration of $2 \mathrm{~min}$, the lowest amplitude setting of $20 \%$, and an ultrasonication period of $4 \mathrm{~s}$ at a $50 \%$ duty cycle were determined as the optimal conditions in this study.

Genetics and Molecular Research 15 (3): gmr.15038988 


\section{ACKNOWLEDGMENTS}

Research supported by the National Natural Science Foundation of China (\#81000427 and \#81370024).

\section{REFERENCES}

Jambrak AR, Mason TJ and Lelas V (2008). Effect of ultrasound treatment on solubility and foaming properties of whey protein suspensions. J. Food Eng. 86: 281-287. http://dx.doi.org/10.1016/j.jfoodeng.2007.10.004

Brenner AJ and Harris ED (1995). A quantitative test for copper using bicinchoninic acid. Anal. Biochem. 226 : 80-84. http://dx.doi.org/10.1006/abio.1995.1194

Gu M, Chen XX, Lin Y, Tian Y, et al. (2010). In vitro antimicrobial efficacy of root canal lubricant and sodium hypochlorite. J. Oral Sci. Res. 25: 462-464.

He YH, Tian XB, Wan HC, Wen YL, et al. (2009). Study on protein extraction methods for Streptococcus mutans. Hua Xi Kou Qiang Yi Xue Za Zhi 27: 100-103.

Nair PN (2006). On the causes of persistent apical periodontitis: a review. Int. Endod. J. 39: 249-281. http://dx.doi. org/10.1111/j.1365-2591.2006.01099.x

NanoDrop (2009). 2000/2000c. Spectrophotometer V1.0 User Manual, Thermo Fisher Scientific Inc.

NanoDrop Technical Support Bulletin T010 (2007). Protein Measurements on NanoDrop Spectrophotometers. NanoDrop Technologies, Inc. Rev, 2007, 5/07.

Saber Sel-D and El-Hady SA (2012). Development of an intracanal mature Enterococcus faecalis biofilm and its susceptibility to some antimicrobial intracanal medications; an in vitro study. Eur. J. Dent. 6: 43-50.

Siqueira Jr JF and Rôças IN (2008). Clinical implications and microbiology of bacterial persistence after treatment procedures. J. Endod. 34: 1291-1301.e3. http://dx.doi.org/10.1016/j.joen.2008.07.028

Smith PK, Krohn RI, Hermanson GT, Mallia AK, et al. (1985). Measurement of protein using bicinchoninic acid. Anal. Biochem. 150: 76-85. http://dx.doi.org/10.1016/0003-2697(85)90442-7 